\title{
Prevalence of syphilis infection in Mozambican women with second trimester miscarriage and women attending antenatal care in second trimester
} Ann Lindstrand, Staffan Bergström, Antonio Bugalho, Giovanni Zanconato,
Anna-Maria Helgesson, Bengt Hederstedt

\begin{abstract}
Objectives-To elucidate whether recent syphilis infection is significantly more prevalent among women with midtrimester miscarriage than among antenatal care attenders in midtrimester pregnancy.

Design-Two categories of pregnant women were compared regarding serological signs of syphilis. Rapid Plasma Reagin (RPR) analyses were done in Mozambique and Veneral Disease Research Laboratory (VDRL) tests in Sweden. In case of RPR and/or VDRL positivity, Treponema pallidum haemagglutination (TPHA) and Captia Syphilis$M$ were performed.
\end{abstract}

Setting-A suburban antenatal care clinic and the emergency ward at the Department of Obstetrics and Gynecology at the Central Hospital in Maputo, Mozambique, were studied June-August 1991.

Subjects-Randomly selected women seeking antenatal care in midtrimester pregnancy $(N=202)$ were compared with 114 women consecutively entering with clinical signs of midtrimester miscarriage.

Department of Gynecologi, Akademiska Hospital, S-751 85, Uppsala, Sweden

$S$ Bergström

A Lindstrand

Department of

Obstetrics and

Gynecology, Ullevå

Hospital, N-0407 Oslo, Norway

$S$ Bergström

Department of

Obstetrics and

Gynecology, Central

Hospital, Maputo,

Mozambique

A Bugalho

$S$ Bergström

G Zanconato

Clinica Obstetrica,

Borgo Roma, 371 00,

Verona, Italy

G Zanconato

The National

Bacteriological

Laboratory, S-105 21,

Stockholm, Sweden

A-M Helgesson

B Hederstedt

Address correspondence to: Dr Ann Lindstrand

Accepted for publication 17 August 1993
Results-Among antenatal care attenders, 37/202 (18.3\%), and among women with midtrimester miscarriage, 37/114 $(32 \cdot 5 \%)$, had syphilis confirmed with the Treponema pallidum haemagglutination test $(p<0.01)$. Significant titres of IgM antibodies tended to be more prevalent among women with miscarriage (7.0\%) than among women attending antenatal care $(4 \cdot 5 \%)$, though the difference only approached statistical significance.

Conclusion-The findings suggest a potential association between syphilis seropositivity and midtrimester miscarriage. Present findings justify more extensive studies to establish whether or not recent syphilis infection is a risk factor for midtrimester miscarriage.

(Genitourin Med 1993;69:431-433)

\section{Introduction}

In developing countries syphilis is still a major threat to reproductive health and one of the major causes of adverse pregnancy outcome. ${ }^{1}$ Prevalences of syphilis seroreactivity in pregnant women attending antenatal clinics in Africa have been reported in the range from $4 \%$ to $15 \%$, but more commonly over $10 \% .^{2}$ With a level of syphilis prevalence in this range, it has been estimated that, owing to syphilis, $5 \%-8 \%$ of all pregnancies surviving past 12 weeks will end in spontaneous abortion, perinatal or infant death, or a living infant with congenital syphilis. ${ }^{2}$

Syphilis may be transmitted to the fetus by the infected mother throughout pregnancy, but fetal lesions develop only after the fourth month in utero when fetal immunological competence becomes established, as reported by Harter et al. ${ }^{3}$ It is generally agreed that the severity of congenital syphilis is related to the time of maternal infection. This severity is determined by the number of Treponema pallidum infecting the fetus and the capacity of the immune response of the mother. If the mother is infected late in pregnancy, infection is known to be more severe because treponaemia will be at its height and the humoral response of the mother is in its early development. ${ }^{3}$ If the mother, on the other hand, is infected before conception or very early in pregnancy, the maternal immune response is effective in clearing most though not all of the organisms, and conceptuses infected under these circumstances are considered to have comparatively mild infection. ${ }^{3}$

Whilst congenital syphilis is a rare phenomenon in the affluent world, it is still a reproductive health problem in impoverished countries. ${ }^{4}$ In one study in Ethiopia, $6 \%$ of the perinatal mortality was attributed to congenital syphilis, ${ }^{5}$ whilst in Zambia $8.6 \%$ of infants examined in a referral hospital had congenital syphilis. ${ }^{6}$

In Mozambique a number of studies have been carried out during the last decade, indicating that pregnancy syphilis represents an important cause of stillbirth. ${ }^{78}$ Prevalence studies from 1983 on antenatal care attenders in Maputo have revealed that up to $9 \%$ bear serological signs of syphilis infection'. More than $90 \%$ of pregnant women in the Maputo city are estimated to attend antenatal care.

Within WHO's Maternal Health and Safe Motherhood Programme a focused interest on the need for effective antenatal care, including syphilis screening and treatment, has been stressed. ${ }^{10}$ They have urged further research on maternal morbidity and effectiveness of care during pregnancy.

The purpose of this study was to elucidate 
whether recent syphilis is significantly more prevalent among women with midtrimester miscarriage than among antenatal care attenders in midtrimester of pregnancy.

\section{Materials and methods}

The study was performed during June to August 1991 and comprised two categories of women in midtrimester pregnancy: firstly, women with clinical signs of miscarriage, and secondly, women with normal pregnancy seeking antenatal care.

From the outpatient gynaecology ward in the Maputo Central Hospital all 114 women entering with midtrimester miscarriage were included in the study (table). Criteria for midtrimester miscarriage were: (a) vaginal bleeding from a pregnant women with estimated gestational length corresponding to 13-26 weeks, (b) fetal tissue expelled through the cervix.

From a suburban antenatal clinic, called Primeiro de Maio, 202 women in midtrimester pregnancy entered the study (table). They were consecutively recruited on their first or second visit before the serology for syphilis was performed.

The antenatal care attenders served as a reference population for the group with midtrimester miscarriage. They were followed by regular contact with an obstetrician until delivery in order to record pregnancy outcome and routines for treatment of syphilis.

Venous blood was collected from the women in both groups. After centrifugation sera were taken for RPR analyses at the Department of Microbiology in Maputo. Serum aliquots were also frozen at $-20^{\circ} \mathrm{C}$ for subsequent transport to Sweden for further analyses at the National Bacteriological Laboratory, Stockholm. Before testing, sera were inactivated at $56^{\circ} \mathrm{C}$ for 30 minutes. In Sweden, VDRL was performed on all sera from the women. In case of RPR and/or VDRL positivity, TPHA and Captia Syphilis$M$ were performed. Captia Syphilis- $M$ is a specific test for Treponema pallidum immunoglobulin $M$ (IgM) with a sensitivity of $94 \%$ for primary syphilis, $85 \%$ for secondary syphilis and $82 \%$ for early latent syphilis, compared with that of the $19 \mathrm{~S}(\mathrm{IgM})$ FTAABS test. ${ }^{11}$ It is an enzyme linked immunoassay, which is a simple and rapid method, with the results being read objectively, an advantage over the laborious and timeconsuming FTA-ABS-test.

Reagents from the Swedish National Bacteriological Laboratory were used for the Veneral Disease Research Laboratory

Table Obstetrical characteristics of the women in the miscarriage group and the antenatal group

\begin{tabular}{|c|c|c|c|c|}
\hline Group of women & Primiparas & $\begin{array}{l}\text { Grand } \\
\text { Multiparas } \\
\geqslant 6\end{array}$ & $\begin{array}{l}\text { Average } \\
\text { gestational } \\
\text { length } \\
\text { (weeks) }\end{array}$ & $\begin{array}{l}\text { Average } \\
\text { no. of } \\
\text { pregnancies }\end{array}$ \\
\hline $\begin{array}{l}\text { Miscarriage-group }(n=114) \\
\text { Antenatal care-group }(n=202)\end{array}$ & $\begin{array}{l}20(17 \cdot 5 \%) \\
33(16 \cdot 2 \%)\end{array}$ & $\begin{array}{ll}10 & (8 \cdot 8 \%) \\
22 & (10 \cdot 8 \%)\end{array}$ & $\begin{array}{l}17 \cdot 2 \\
19 \cdot 3\end{array}$ & $\begin{array}{l}3 \cdot 5 \\
3 \cdot 1\end{array}$ \\
\hline
\end{tabular}

(VDRL) test. Treponema pallidum haemagglutination assay (TPHA) (Fujirebio Inc, Tokyo, Japan), Captia Syphilis-M (Mercia diagnostics Ltd, Shalford, Surrey, England) and Rapid Plasma Reagin (RPR) (bioMerieux, Chardonniére-les-Bains, Cedex, France) were performed according to the manufacturers' descriptions.

Statistical analyses were performed using the chi square test. $95 \%$ prevalence confidence intervals were calculated.

\section{Results}

The obstetrical characteristics of the two groups are presented in the table. The proportion of women with seropositive IgM test was $4.5 \%(9 / 202)$ with $95 \%$ confidence interval (C.I.) $1 \cdot 6-7 \cdot 4 \%$, among the antenatal care attenders and $7.0 \%(8 / 114)$ with $95 \%$ C.I. $2 \cdot 3-11 \cdot 7 \%$, among the miscarriage group. The difference is not statistically significant. If all women in both groups are compared regarding the proportions with seropositive syphilis (including those being IgM-positive and IgM-negative), $18.3 \% \quad(37 / 202)$ with 95\% C.I. $12 \cdot 9-23 \cdot 7 \%$ antenatal attenders and $32.5 \%(37 / 114)$ with $95 \%$ C.I. $23 \cdot 9-41 \cdot 1 \%$ women with miscarriage were syphilis seropositive. The difference is significant $\left(\chi^{2}=7.4 ; p<0.01\right)$.

In the total group of 202 women attending for antenatal care, $87 \%$ (175) were followed past the second trimester and $70 \%$ (142) were followed to delivery. One of the 165 seronegative women had a stillbirth, but the rest had normal pregnancy outcomes.

Of the 37 women with serological signs of syphilis in the antenatal care group, 26 were successfully followed to delivery. Two of the women with serology indicative of active syphilis in the midtrimester had stillbirths, though one had received adequate treatment. Five women were followed with normal pregnancies past the midtrimester, but were lost later on. Six women never turned up at the antenatal clinic again. Of these six, two had serological signs of active syphilis at their first visit to the antenatal clinic.

Nineteen of the 37 women with seroreactive syphilis were treated according to common practice with benzathine penicillin. Six women did not return for a second visit to the antenatal clinic and two women were falsely negative in their syphilis diagnosis in Maputo. This leaves 10/37 (27\%) women, with locally confirmed seroreactive syphilis, who did not get treatment.

\section{Discussion}

It is generally agreed that syphilis is of limited importance in the etiology of first trimester miscarriage. ${ }^{3}$ In third trimester pregnancy wastage syphilis is considered a more significant aetiological factor. ${ }^{1} 257$ Midtrimester pregnancy has attracted little attention in the literature as far as syphilis is concerned. One study on 240 women with spontaneous abortion in Zambia showed a TPHA-confirmed 
syphilis prevalence of $18 \cdot 8 \% .^{12}$ Of these abortions, $72 \%$ occurred before the 20th week. The present study was initiated with the intention to elucidate whether or not syphilis plays a significant etiological role in midtrimester miscarriage in a society severely affected by pregnancy syphilis. ${ }^{8}$

Syphilis-IgM antibodies tended to be more prevalent among midtrimester miscarriage women $(7.0 \%)$ than among midtrimester antenatal attenders ( $4 \cdot 5 \%)$. Since serological signs of syphilis (old and recent combined) were more prevalent among women with midtrimester miscarriage than among normal midtrimester antenatal attenders, it may be assumed that the miscarriage group was more exposed to risk of syphilis infection. The sample size may have been too limited to confirm a difference in the prevalence of recent syphilis infection.

All gynaecologic emergencies in Maputo are referred to the Central Hospital. In this hospital mostly high risk antenatal clients, or pregnant women referred due to disease, are seen. There is no ideal referent group, representing uneventful pregnancies, available within the hospital, but the one seen in the antenatal clinic would seem the least biased one.

The inclusion criteria in the miscarriage group was clinically confirmed miscarriage and denial of an induced abortion by the women. The availability of legal abortions in Maputo would tend to make the number of illegal ones much more limited than in a setting without this legal facility. This is, however, a difficult problem and it may well be that a (presumably small) proportion of these "miscarriages" in fact had been induced.

Yaws, the non-veneral treponematosis, can give falsely positive results when syphilis is serologically tested for. WHO reported in 1982 that no yaws infection existed in Mozambique. ${ }^{13}$ There is no evidence reported of any resurgence of yaws since then. It does not seem plausible that yaws would have interfered with the results of the present study.

Though this was a study population, $27 \%$ of the women with locally diagnosed seroreactive syphilis did not receive treatment. Similar findings have been reported in a study from Zambia addressing failures to screen all antenatal care attenders, to treat partners and to detect acquired infection during pregnancy after the initial screening has been done. ${ }^{14}$ One difficulty in Maputo is the frequent lack of antibiotics. Recent results from syphilis screening on parturient women at the Maputo Central Hospital (MCH) show a syphilis seroreactive prevalence of $8 \cdot 8 \% .15$
Most of the women giving birth at the $\mathrm{MCH}$ are referred from antenatal care clinics in the Maputo area, but apparently many of them had not received adequate treatment. These recent data also indicate a statistically significant increase in seroreactive syphilis between $2.8 \%$ in 1985 and $8.8 \%$ in 1991 in women giving birth at the $\mathrm{MCH} .{ }^{15}$ This is in agreement with recent evidence for an increase in congenital syphilis among newborns in Maputo. ${ }^{16}$

In conclusion, the present results suggest a potential association between syphilis seropositivity and midtrimester miscarriage. To our knowledge there are no data on the role of syphilis as an etiologic factor in midtrimester miscarriage in the literature. The present results, therefore, justify more extensive investigation to establish background factors in midtrimester miscarriage.

The financial support from SAREC (Swedish Agency for Research Cooperation in Developing Countries) is gratefully acknowledged. Reagents and testkit for the VDRL and TPHA tests were supplied by the National Bacteriological Laborator (SBL). The collaboration of staff at SBL is gratefully acknowledged.

1 Arya OP, Osoba AO, Bennet FJ. Tropical Venereology Churchill Livingstone, Edinburgh, 1980

2 Schulz KF, Cates W Jr, O'Mara PR. Pregnancy loss, infant death, and suffering: legacy of syphilis and gonorrhoea in Africa. Genitourin Med 1987;63:320-5.

3 Harter CA, Benirschke $\mathrm{K}$. Fetal syphilis in the first trimester. Am ₹ Obstet Gynecol 1976;124:705-11.

4 Mascola L, Pelosi R, Blount JH, Alexander CE, Cates W Jr. Congenital syphilis revisited. Am $¥$ Dis Child 1985;139: Congen

5 Judge DM, Tafari N, Naeye RL. Congenital syphilis and perinatal mortality. Ped Pathol 1986;5:411-20.

6 Chattopadhyay, B. Prevention of congenital syphilis. $\mathrm{Br} F$ Hosp Med 1988;40:68-70.

7 Axemo P, Liljestrand J, Bergström S. Causes of stillbirth in Maputo, Mozambique. In: Liljestrand J. Maternal morbidity in Mozambique (Thesis, Uppsala University, 1985)

8 Hederstedt B, Liljestrand J, Bergström S, Axemo $P$, Nieuwenhuis $\mathrm{F}$. IgM-antibodies against $T$ Pallidum detected in sera from mothers of stillborn babies in Mozambique by the solid-phase hemadsorption assay (SPHA). International foumal of STD and AIDS 1992; 3:347-9.

9 Liljestrand J, Bergström S, Nieuwenhuis F, Hederstedt B. Syphilis in pregnant women in Mozambique. Genitourin Med 1985;61:355-8.

10 Rooney C. Antenatal care and maternal health: How effective is it? A review of the evidence. WHO/MSM 92/4.

11 Lefevre J-C, Bertrand M-A, Bauriaud R. Evaluation of the Captia Enzyme Immunoassays for Detection of Immunoglobins $G$ and $M$ to Treponema pallidum in Syphilis. 于 Clin Microbiol 1990;28:1704-7.

12 Ratman AV, Din SN, Hira SK, et al. Syphilis in pregnant women in Zambia. Br Y Venereol Dis 1982;58:355-8.

13 Treponaemal infection. Report of a WHO scientific group. World Health Organisation, Geneva. Technical group. World Health Organisation,

14 Hira SK, Bhat GJ, Chicamata DM. Syphilis intervention in pregnancy: Zambian demonstration project. Genito urin Med 1990;66:159-64

15 Lindstrand A, Bergström S, Bugalho A, Helgesson A-M Hederstedt B. Perinatal transmission of parturien syphilis in Maputo, Mozambique Gyn Obstet Invest (In press).

16 Antonio C, Davanzo R. Congenital syphilis in Mozambique: the diagnostic complementary role of laboratory and radiological investigation. Centr Afr $\mathcal{F}$ Med 1989;35: 552-5. 